\title{
Light source for stray light characterisation of EnMAP spectrometers
}

\author{
Charles Pope and Andreas Baumgartner \\ DLR, Remote Sensing Technology Institute, Oberpfaffenhofen, D-82234 Wessling, Germany
}

\begin{abstract}
EnMAP is a German hyperspectral satellite mission. The Remote Sensing Technology Institute of the German Aerospace Center is responsible for the stray light characterisation of the satellite's spectrometers. This paper describes the light source developed for that purpose and how it will be employed to characterise the in-band, in-field stray light. This type of stray light can be characterised by measuring the point spread function (PSF) at multiple locations on the detector. Because of the cumulative effect of stray light, it is necessary to characterise the PSF in the near- and far-field. The PSF can span a range of more than 6 orders of magnitude from the peak to the wings at the edges of the detector, exceeding the intrinsic dynamic range of the instrument. Therefore a high dynamic range (HDR) PSF measurement is required: A spectrally and angularly narrow spot of light is guided onto the instrument then measurement frames of multiple signal levels are acquired. The signal level is controlled by changing the instrument's integration time or changing the neutral density filter of the light source. The overexposed pixels in the higher signal level frames are excluded, then the multiple image frames are combined into one HDR frame which can then be used in the stray light correction algorithm. This HDR 'bracketing' technique is demonstrated here with DLR's HySpex VNIR-1600 airborne imaging spectrometer.
\end{abstract}

Keywords: Stray light, hyperspectral imaging spectrometer, characterisation, light source, HySpex, EnMAP

\section{INTRODUCTION}

The Environmental Mapping and Analysis Program (EnMAP) is a German satellite mission to monitor Earth's environment and ecosystems on a global scale. ${ }^{1}$ The satellite, built by OHB-System AG, will carry two hyperspectral imaging spectrometers sensitive to the VNIR and SWIR spectral range. An important component of the pre-launch characterisation and calibration campaign is the stray light characterisation. Key challenges of stray light measurements are achieving a high dynamic range signal, while keeping the spectral and spatial stray light low in the measurement setup. This paper explains the stray light characterisation methodology and the light source that is being developed at the Remote Sensing Technology Institute (IMF) of the German Aerospace Center (DLR) in Oberpfaffenhofen for the characterisation measurements.

Here, a general overview is given of the stray light and its measurement. In Sec. 2, specifications of the light source are presented and the details of the measurement setup are given. In Sec. 3, the measurement procedure is described in detail. Finally, in Sec. 4 the data processing is discussed using measurement results obtained with DLR's HySpex VNIR-1600² imaging spectrometer for illustration.

\subsection{Stray Light Definition}

Stray light, as the name suggests, is unwanted light on the detector array of an optical system. Stray light can originate from several sources: scattering from imperfect optical surfaces, specular reflections, diffraction effects at edges, etc. For a full overview of different types of stray light see Ref. 3. Here, we are only concerned with measuring diffuse, in-band, in-field stray light. That is, the fraction of stray light which originates from nominal signal which falls on the detector array. It can therefore be corrected, since a transfer function can be determined between the measured signal and its stray light component. Diffuse refers to the fact we are not considering focused reflections. In-band refers to light that is within the spectral range of the detector and in-field means light within the geometric field of view of the input optics.

Further author information: (Send correspondence to A. Baumgartner)

A. Baumgartner: E-mail: Andreas.Baumgartner@DLR.de 
If a point light source was directed at an imaging spectrometer, a characteristic point spread function (PSF) would be measured on the detector (see Fig. 1). The area outside of the peak of the PSF is what we define to be the stray light. The exact definition of the border between intended signal and stray light is partially arbitrary. Often stray light performance of an instrument will be specified as a percentage of the intended (in-band, infield) signal. So changing the definition of this region size will of course affect the apparent stray light in the instrument, therefore one must consider definitions carefully when specifying stray light performance. Ref. 3 describes how varying the definition of this region will affect the fraction of stray light calculated.

When measured in isolation like this, the stray light signal is very weak, but it can be significant when its cumulative effect is considered, particularly on high contrast scenes, where dark areas can be strongly effected by stray light.

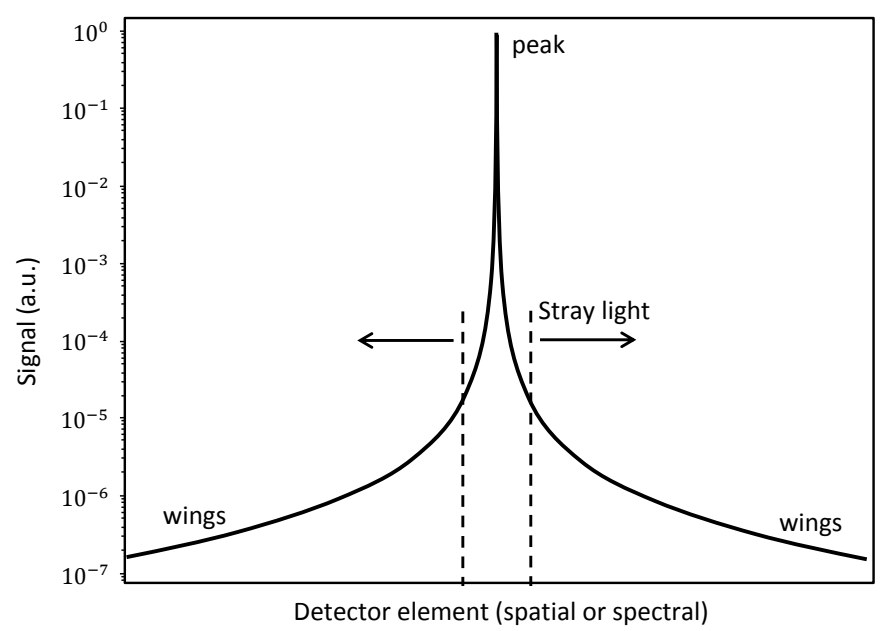

Figure 1. One dimensional slice through a conceptual PSF.

\subsection{Stray Light Measurement}

Characterisation of in-band, in-field stray light involves guiding a collimated, spectrally narrow light spot onto the spectrometer's input optics. As mentioned, this results in a PSF on the detector array. The difference of the signal from the peak to the wings of the PSF can easily exceed the dynamic range of the detector, thus a 'bracketing' technique must be used. ${ }^{4}$ In this technique, detector frames are recorded at two or more signal levels and then combined to create a single high dynamic range (HDR) characterisation frame. For example, one frame is recorded at a nominal signal level, the peak signal of the PSF must be below the saturation level. The signal is then increased (by increasing the integration time for example) and a second frame is taken. In this second frame the signal level at the peak of the PSF is now in saturation and unusable, but the signal in the wings has improved SNR. The saturated data is discarded, and these two frames are then combined to form the HDR PSF characterisation frame. Practically, it is often required to use more than just two signal levels to ensure no gaps in the combined frame. This is because the steep slope of the PSF edges rapidly cover several orders of magnitude, coupled with the fact that some detector elements could also be effected by blooming and are unusable. Thus the number of signal levels is highly dependent on the shape of the PSF being measured.

These HDR characterisation frames will then be used to build a stray light correction matrix, which is used in the stray light correction algorithm. The matrix describes how the light falling on each detector element spreads onto all the other detector elements. The number of elements in a full resolution stray light correction matrix is equal to the number of detector elements squared. Therefore, to save processing effort, a binning scheme is intended to be used for the stray light correction of EnMAP.

If a binning scheme is used, then it is not necessary to measure the true point spread function for each detector element. Instead, a larger illumination spot may be used (spectrally and spatially wider than a single 
detector element), which also has the advantage of providing more light to the detector thus improving the signal to noise ratio (SNR) of the measurements. This lower resolution spread function will still be referred to as the PSF from here on for simplicity.

\section{MEASUREMENT SETUP}

\subsection{Light Source}

The core of the measurement setup is the light source. In order to characterise in-band, in-field stray light, DLR has developed the Stray Light Test Source (SLTS), it is described here.

\subsubsection{Design}

The SLTS consists of an Energetiq EQ-99XFC ${ }^{5}$ laser driven plasma lamp, two custom-made filter wheels, a shutter and two parabolic mirrors (see Fig 2).

A light beam exits the plasma lamp through an optical fibre and is collimated by an off-axis parabolic mirror (OAPM). Harmful UV-light below $320 \mathrm{~nm}$ is suppressed by a long pass filter. A neutral density (ND) filter in the first wheel sets the light level. The beam then passes through a band pass filter housed in the second wheel and is re-coupled into an output fibre with a second OAPM. A mechanical shutter is installed in the beam path so that the output can be switched on or off.

The first wheel has 16 places available for ND filters, while the second wheel holds a set of band pass filters, 11 in the VNIR wavelength range and 4 in the SWIR wavelength range. The band pass filters' centre wavelengths were chosen to uniformly span the spectal range of the EnMAP spectrometers, while avoiding atmospheric absorption bands. The filters are stacked in order improve out of band suppression and edge steepness.

The SLTS can be controlled over a serial interface. This allows the remote selection of band pass filter, ND filter and shutter position. With this control scheme, it is very easy to integrate the SLTS into any automated measurement setup.

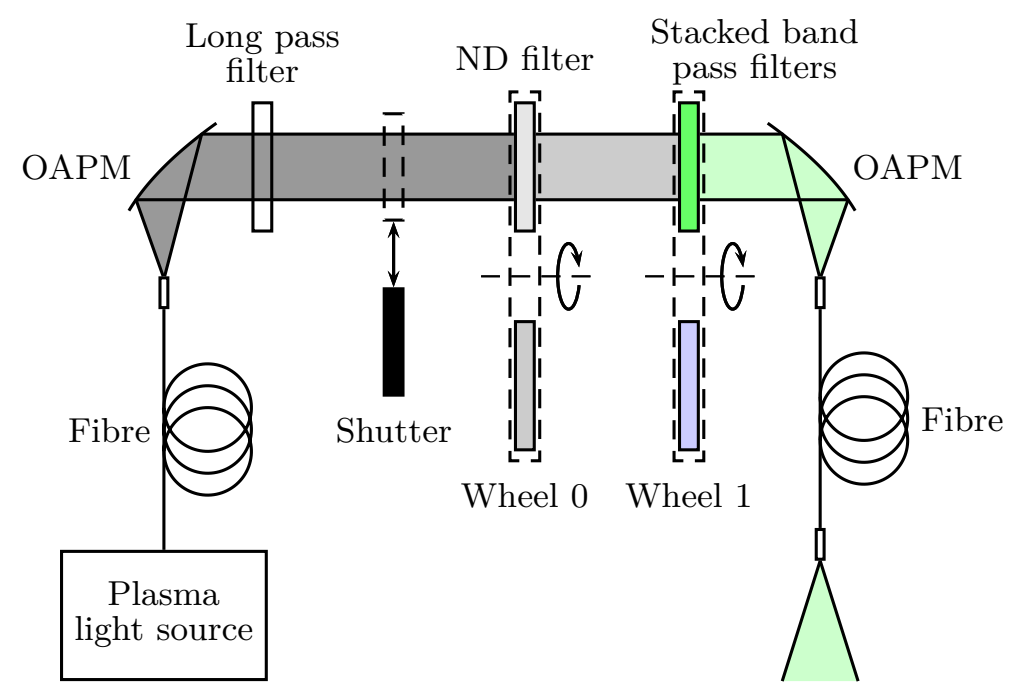

Figure 2. Stray Light Test Source, optical configuration. OAPM $=$ off-axis parabolic mirror.

\subsubsection{Specifications}

The SLTS must meet several requirements to be useful for stray light characterisation. Firstly, a high radiance light source is required to make the weak stray light signal visible. The output must have a high spectral purity $\left(10^{-9}\right.$ out-of-band suppression). The transition between the pass band and block band should have steep edges. The SLTS shall be stable over the duration of measurement. The light source uses the Energetiq EQ-99XFC 
laser driven plasma lamp for its high radiance and stability. Multiple of band pass filters are stacked to achieve the high spectral purity and edge steepness requirement. The characterisation of the light source and filters is detailed in Ref. 6. Table 1 lists some key specifications of the light source.

Table 1. Key specifications of the SLTS. The optical density of several filters has been validated at $660 \mathrm{~nm}$ and $960 \mathrm{~nm}$ using lasers.

\begin{tabular}{lllll}
\hline $\begin{array}{l}\text { Central } \\
\text { wavelength } \\
(\mathrm{nm})\end{array}$ & $\begin{array}{l}\text { Filters } \\
\text { stacked }\end{array}$ & $\begin{array}{l}\text { Min. optical } \\
\text { density outside of } \\
\text { pass band }\end{array}$ & $\begin{array}{l}\text { Width of pass } \\
\text { band }(\mathrm{nm}) @ \\
-80 \mathrm{~dB}\end{array}$ & $\begin{array}{l}\text { Radiant } \\
\text { power } \\
(\mu \mathrm{W})\end{array}$ \\
\hline 450 & 3 & 9 & 25 & 590 \\
488 & 3 & 9 & 33 & 330 \\
532 & 4 & 9 & 25 & 550 \\
590 & 3 & 9 & 38 & 90 \\
635 & 4 & 9 & 25 & 500 \\
660 & 4 & 9 & 25 & 500 \\
730 & 3 & 9 & 30 & 110 \\
780 & 3 & 9 & 25 & 430 \\
810 & 3 & 9 & 25 & 500 \\
850 & 3 & 9 & 25 & 500 \\
905 & 4 & 9 & 45 & 230 \\
1020 & 3 & 9 & 32 & 34 \\
1250 & 2 & 9 & 45 & 316 \\
1600 & 2 & 9 & 39 & 294 \\
2150 & 2 & 9 & 62 & 52
\end{tabular}

\subsection{Light Coupling to Instrument}

To employ the SLTS for stray light measurements, it is required to use additional opto-mechanical components to shape, collimate and guide the beam from the light source to the instrument under test. In the case of the EnMAP characterisation measurements at OHB-System AG, the setup in Fig. 3 is used. The light from the SLTS is coupled into an optical fibre, guided through a fibre mixing system to homogenise the beam, redirected by mirrors, re-sized by a slit (the focal plane target) and collimated. The collimator is mounted on a hexapod actuator that enables the illumination angle of the instrument to be changed. Several different focal plane target sizes are available to shape the beam. Further details of the optical ground support equipment that will be used during the EnMAP characterisation can be found in Ref. 7.

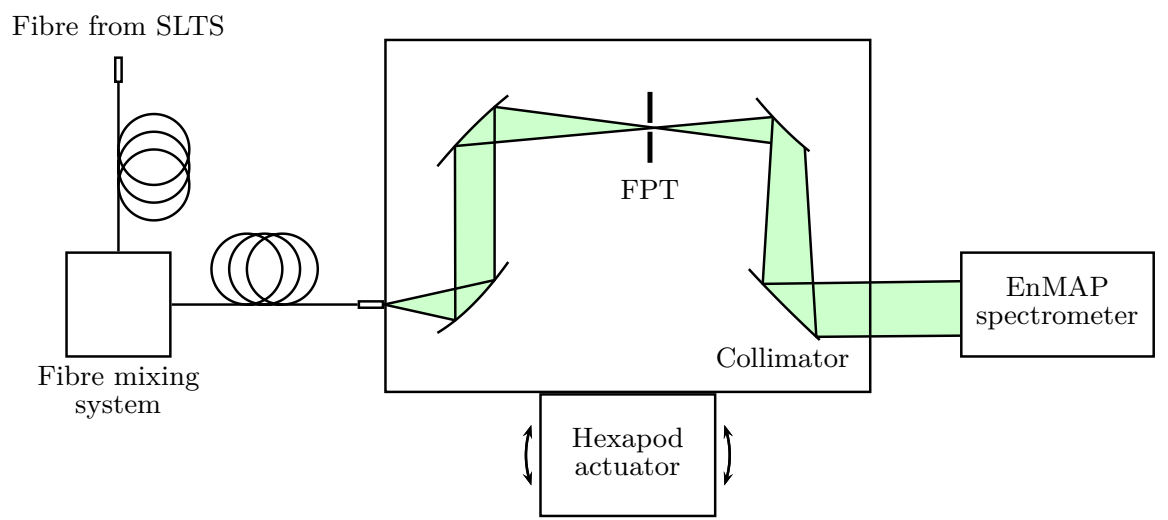

Figure 3. Light coupling setup used in the characterisation of EnMAP spectrometers. The Fibre mixing system (FMS) ensures spatially homogeneous light. The Focal Plane Target (FTP) is a slit of selectable size for reshaping the beam. 
In the case of measurements performed on the HySpex spectrometer at DLR's calibration home base (CHB) facility ${ }^{8}$ the SLTS beam is reshaped by imaging the fibre core onto a slit using two off-axis parabolic mirrors. The slit is located in the focal plane of a collimator. This results in a divergence angle and beam width sufficient to overfill the along-track instantaneous field of view and instrument aperture. Different illumination angles are realised by the rotating, linear moving 'folding' mirror (Fig. 4).

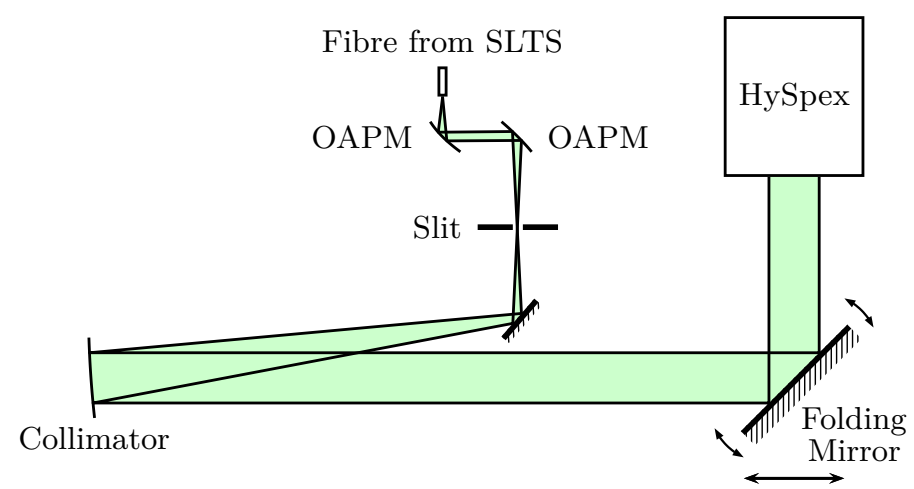

Figure 4. Light coupling setup used in the characterisation of HySpex spectrometers. Two off-axis parabolic mirrors image the core of the fibre coming from the SLTS on a slit. The spatially filtered image is then guided by a collimator over a rotating and linear moving 'folding' mirror to the sensor.

\section{MEASUREMENT METHOD}

The light source is used to measure the PSF of the spectrometer under test. The measurement requires a high dynamic range, so several measurements need to be made at different signal levels. This section describes the determination of the signal levels, the sample grid of measurements and the measurement procedure.

\subsection{Spectral-Spatial Sampling Points}

In a push broom spectrometer such as HySpex or EnMAP one dimension of the detector array represents the spatial axis, and the second, the spectral axis. Characterisation measurements must cover both axes with appropriate sample spacing. The set of spectral positions is already fixed by the light source's band pass filters, which were chosen based on the EnMAP spectrometers sensitive spectral range. There are 11 VNIR and 4 SWIR wavelengths. The sample spacing in the spatial direction across the detector should be set according to how rapidly the PSF changes. A rapidly changing PSF demands a finer sampling of measurement points. For measurements of the HySpex VNIR-1600 instrument, seven spatial positions were considered sufficient. ${ }^{4}$ (Approximately 220 spatial pixels between samples.)

\subsection{Signal Levels}

As described in Sect. 1, the HDR PSF is built up of multiple signal level frames. The set of signal levels that works for one spectrometer may be different to those for another (HySpex vs EnMAP), so a signal ranging test is used to determine the required set of signal levels before the automatic test sequence is executed at all geometric positions.

First, an integration time and ND filter is selected which results in a nominal signal level of the peak of the PSF (close to $100 \%$ linear full well capacity of the detector). From here on, frames are taken at increasing signal levels, up to the limit, increasing half an order of magnitude each step. This is achieved by a combination of stepping down the ND filter attenuation and/or increasing the integration time. Depending on the detector under test, it may be preferable to change ND filters rather than integration time if there are integration time dependent non-linearities. In the case of the EnMAP spectrometer, it is also possible to change the gain setting of the instrument and the size of the FPT (Fig. 3) to increase the signal level. The frames are normalised to account for integration time, ND filter, FPT or gain change and plotted on one another. It can then be quickly 
recognised what the minimum required set of signal levels is by selectively toggling the visibility of each frame while looking for gaps in the PSF. For example, the signal ranging test of the HySpex PSF measured with the 730 $\mathrm{nm}$ band pass filter gathered frames at signal levels: nominal, $5 \times, 10 \times, 50 \times, 100 \times, 500 \times, 1000 \times, 5000 \times, 10000 \times$. Figure 5 shows that for this PSF, the signal sub set of: nominal, $5 \times, 50 \times, 500 \times, 10000 \times$ is sufficient to build an HDR PSF with no gaps in the centre.

Note that the HySpex PSF in Fig. 5 is measured over approximately 6.4 orders of magnitude: 2.4 orders (instrument linear dynamic range) +4 orders (max signal frame). In the most conservative estimate, a measurement of 8 orders of magnitude would be required so that stray light correction algorithm can reduce stray light errors to insignificant levels. However for EnMAP, preliminary estimates show it will be possible to measure over a dynamic range of at least than 8 orders of magnitude. This is due to the sensitivity of the detector, combined with the possibility of increasing the signal level using the FPT and gain settings. Thus it is expected there will be few to no gaps in the PSF far field, remaining gaps will be irrelevant for the stray light correction.

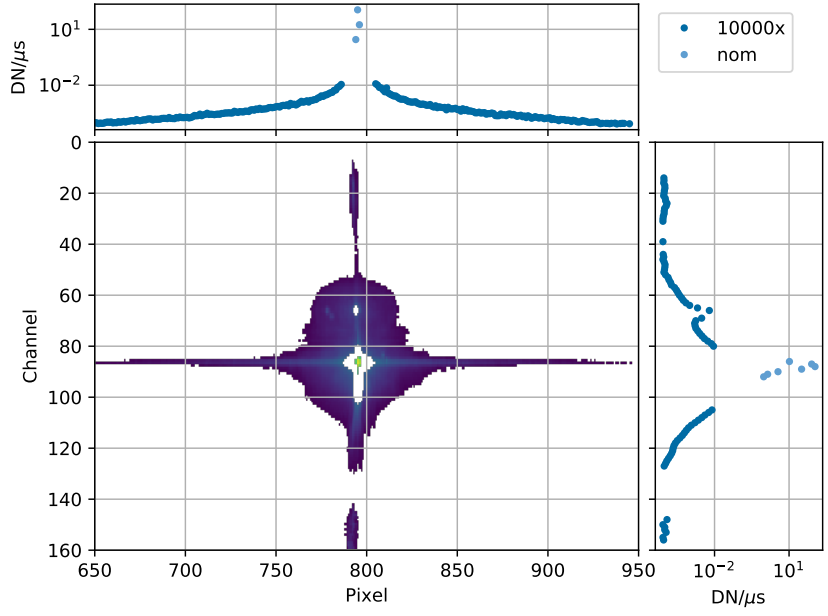

(a) Two signal levels

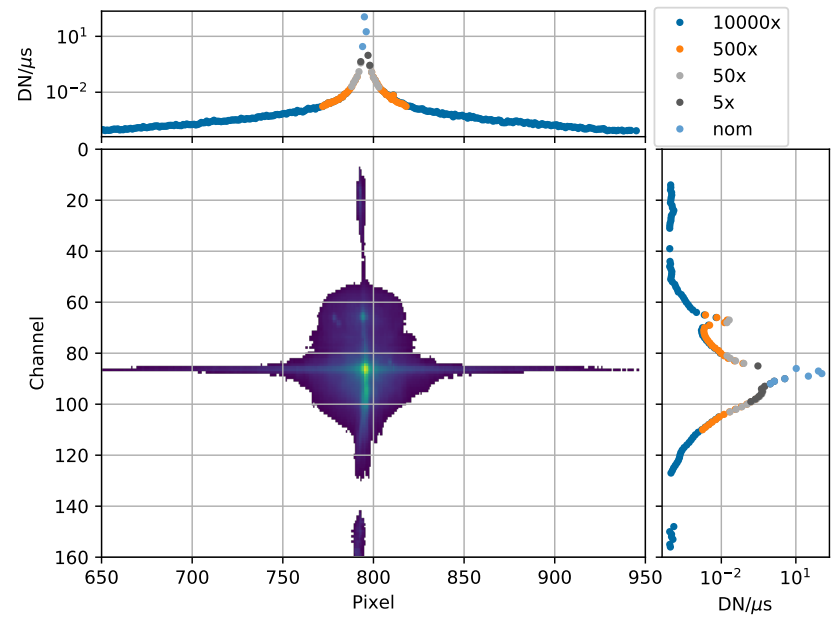

(b) Five signal levels

Figure 5. Example of a high dynamic range point spread function of HySpex VNIR-1600. (a) using just the nominal and maximum signal level frames. (b) using additional signal levels to fill the gaps. Note the 'shoulder' in the spectral direction above channel 90 is caused by a filter installed to block higher grating orders. The small feature around channel 65 is a focused reflection whose geometric position varies with illumination angle (this would not be considered 'diffuse' stray light).

\subsection{Procedure}

The typical measurement procedure with the HySpex at DLR is straight forward. After allowing sufficient warmup time for the plasma light source and instrument, the spatial position of the illumination is set by adjusting the position of the folding mirror (see Fig. 4). Next the band pass filter of the light source is set, along with the required ND filter and integration time. Multiple frames are recorded. The light source shutter is then closed and several background frames are recorded. This process is repeated by an automatic script for all the required signal levels and spectral-spatial positions. 


\section{DATA PROCESSING}

A data processing program was developed to assist in creating the HDR characterisation frames from the multiple signal level 'bracketing' frames. The scheme is similar to that in Ref. 9. A visualisation is shown in Fig. 6. The steps in the processing are:

1. The data is corrected for non-linearity.

2. Outliers in the time domain are filtered out. (multiple frames are taken, at each setting, if a detector element in a frame is outside 4 standard deviations of the mean, it is considered an outlier and excluded from the subsequent analysis by setting it to NaN.)

3. The average background frame is subtracted from the average measurement frame.

4. Detector elements that are saturated are detected (using the data before it was averaged) and set to NaN, along with a margin of neighbouring detector elements, that are effected by blooming. The size of this exclusion region is specific to the detector being characterised.

5. Signal limits are considered. There may be maximum or minimum signals that are acceptable due to limits of the linearity correction. Detector elements outside of the max-min range are set to NaN. E.g. with the HySpex signals below 10 DN (after background subtraction) are considered too low.

6. SNR ratio is also used to filter out bad data. Detector elements that have a SNR below the nominated threshold are set to $\mathrm{NaN}$

7. The frames must be normalised by the integration time and transmission factor of the ND filter (if any were used).

8. The frames are combined by taking an average weighted by the SNR. NaN values do not count towards the average.
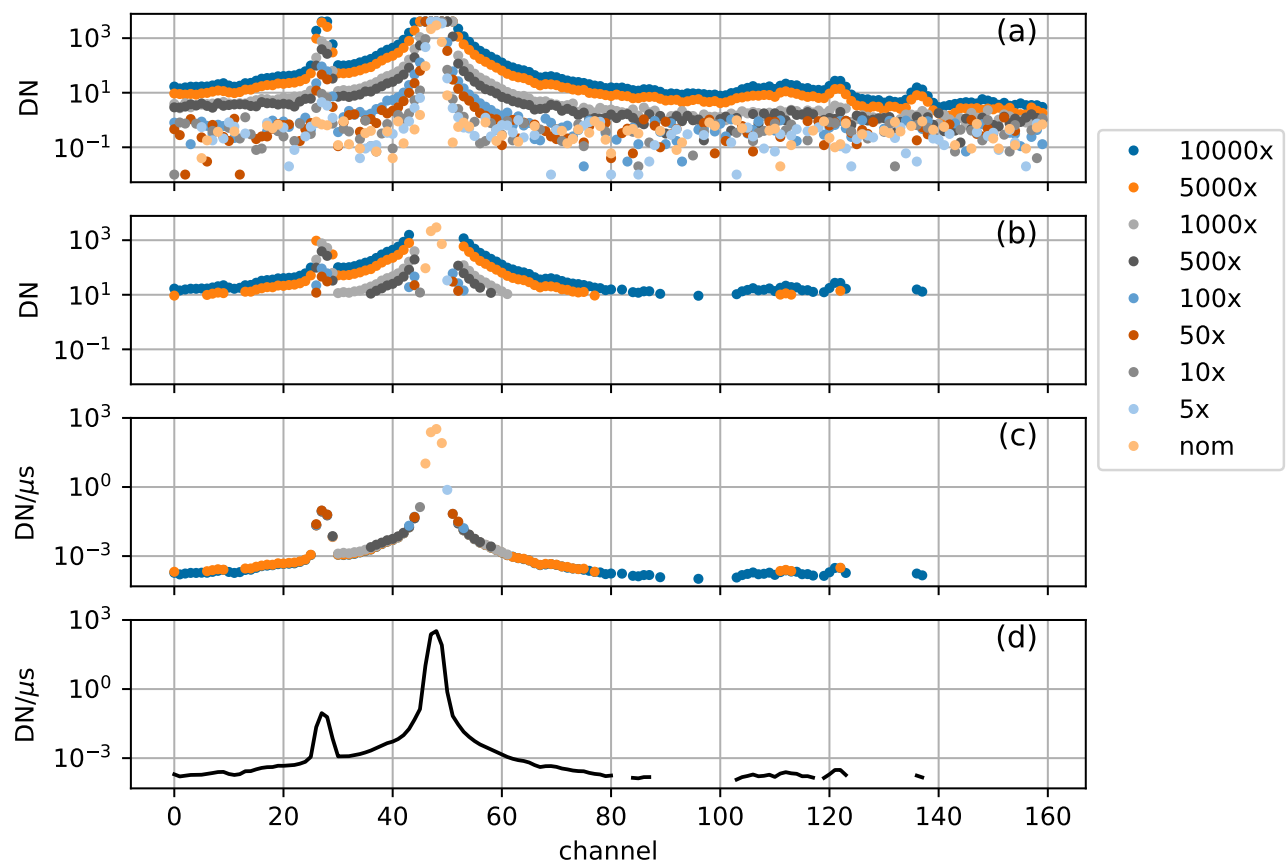

Figure 6. Processing steps visualised on the spectral axis (HySpex data). Colour represents signal level relative to nominal. Signal is in digital numbers (DN) or DN per unit time. (a) steps 1-3, (b) steps 4-6, (c) step 7 (d) step 8 . The dynamic range is extended from about 2 orders of magnitude in (b) to slightly over 6 orders of magnitude in (d). 


\section{CONCLUSION}

A light source suitable for in-band, in-field stray light characterisation has been constructed and tested at DLR. Estimates show that the light source will be sufficient to measure the PSFs of the EnMAP spectrometers with a high dynamic range when it is employed during the pre-launch characterisation campaign. DLR's HySpex VNIR-1600 instrument was used to develop the data collection and processing methodology. A processing chain for creating high dynamic range characterisation frames is under development. This processing chain will be applied to the EnMAP stray light characterisation data.

\section{ACKNOWLEDGMENTS}

The authors would like to acknowledge the work of J. Brachmann \& M. Kretz for the design \& characterisation of the stray light test source. In addition: L. Polz, C. Köhler, P. Haschberger \& P. Gege for giving helpful feedback.

The development of the stray light characterisation \& correction method for the EnMAP instrument is made for the EnMAP prime contractor OHB System AG who is under contract to DLR with funding from the German Federal Ministry of Economic Affairs and Energy (50 EP 0801).

\section{REFERENCES}

[1] Guanter, L., Kaufmann, H., Segl, K., Foerster, S., Rogass, C., Chabrillat, S., Kuester, T., Hollstein, A., Rossner, G., Chlebek, C., Straif, C., Fischer, S., Schrader, S., Storch, T., Heiden, U., Mueller, A., Bachmann, M., Mühle, H., Müller, R., Habermeyer, M., Ohndorf, A., Hill, J., Buddenbaum, H., Hostert, P., van der Linden, S., Leitão, P., Rabe, A., Doerffer, R., Krasemann, H., Xi, H., Mauser, W., Hank, T., Locherer, M., Rast, M., Staenz, K., and Sang, B., "The enmap spaceborne imaging spectroscopy mission for earth observation," Remote Sensing 7(7), 8830-8857 (2015).

[2] Koehler, C., "Airborne imaging spectrometer hyspex," Journal of large-scale research facilities JLSRF 2, A93 (2016).

[3] Kleipool, Q., Ludewig, A., Babić, L., Bartstra, R., Braak, R., Dierssen, W., Dewitte, P.-J., Kenter, P., Landzaat, R., Leloux, J., Loots, E., Meijering, P., van der Plas, E., Rozemeijer, N., Schepers, D., Schiavini, D., Smeets, J., Vacanti, G., Vonk, F., and Veefkind, P., "Pre-launch calibration results of the tropomi payload on-board the sentinel-5 precursor satellite," Atmospheric Measurement Techniques 11(12), 6439-6479 (2018).

[4] Lenhard, K., Baumgartner, A., Gege, P., Nevas, S., Nowy, S., and Sperling, A., "Impact of improved calibration of a neo hyspex vnir-1600 sensor on remote sensing of water depth," IEEE Transactions on Geoscience and Remote Sensing 53(11), 6085-6098 (2015).

[5] Energetiq, "EQ-99XFC LDLS Compact, Long-Life, High-Brightness, Broadband LaserDriven Light Source with Fiber-Coupled Output." https://www.energetiq.com/ fiber-coupled-laser-driven-light-source-long-life-compact. (Accessed: 8 August 2019).

[6] Kretz, M., Design and construction of a light source setup for stray light measurements in array spectrometers, Master's thesis, KIT Karlsruhe (1 2018).

[7] Lettner, M., Lamour, T., Polz, L., Glastre, W., Compain, E., and Fischer, S., "Versatile full aperture illumination ogse setup for alignment and end-to-end calibration of the enmap hyperspectral imager," in [International Conference on Space Optics - ICSO 2018], (7 2019).

[8] Brachmann, J., Baumgartner, A., and Gege, P., "The calibration home base for imaging spectrometers," Journal of large-scale research facilities 2, A82 (2016).

[9] Kouremeti, N. and Groebner, J., "Report on bandpass and line-spread function and determination of straylight matrix," tech. rep., Physikalisch-Meteorologisches Observatorium Davos World Radiation Center (2017). 